\title{
Silencing of genes responsible for polyQ diseases using chemically modified single-stranded siRNAs
}

\author{
Agnieszka Fiszer ${ }^{\bowtie}$, Marianna E. Ellison-Klimontowicz and Wlodzimierz J. Krzyzosiak ${ }^{\bowtie}$ \\ Department of Molecular Biomedicine, Institute of Bioorganic Chemistry, Polish Academy of Sciences, Poznań, Poland
}

\begin{abstract}
Polyglutamine (polyQ) diseases comprise a group of nine genetic disorders that are caused by the expansion of the CAG triplet repeat, which encodes glutamine, in unrelated single genes. Various oligonucleotide (ON)-based therapeutic approaches have been considered for polyQ diseases. The very attractive CAG repeat-targeting strategy offers selective silencing of the mutant allele by directly targeting the mutation site. CAG repeat-targeting miRNA-like siRNAs have been shown to specifically inhibit the mutant gene expression, and their characteristic feature is the formation of mismatches in their interactions with the target site. Here, we designed novel single-stranded siRNAs that contain base substitutions and chemical modifications, in order to develop improved therapeutic tools with universal properties for several polyQ diseases. We tested these ONs in cellular models of Huntington's disease (HD), spinocerebellar ataxia type 3 (SCA3) and dentatorubral-pallidoluysian atrophy (DRPLA). Selected siRNAs caused the efficient and selective downregulation of the mutant huntingtin, ataxin-3 and atrophin-1 levels in cultured human fibroblasts. We also prove the efficiency of novel ONs, with chemical modification pattern mainly containing 2'-fluoro (2'F), in HD mouse striatal cells.
\end{abstract}

Key words: siRNA, CAG repeats, polyglutamine diseases, Huntington's disease

Received: 30 May, 2016; revised: 28 June, 2016; accepted: 05 July, 2016; available on-line: 21 October

\section{INTRODUCTION}

For fifteen years, RNAi-based strategies designed for mutant gene down-regulation, have been rapidly being developed. A variety of oligonucleotides (ONs) have been tested for polyglutamine (polyQ) diseases (Fiszer \& Krzyzosiak, 2014). This group of neurological disorders is caused by ORF-located CAG repeat expansion in specific genes, and includes HD, DRPLA, spinal bulbar muscular atrophy (SBMA), and SCA types 1, 2, 3, 6, 7 and 17 (Fan et al., 2014).

Although polyQ disorders remain incurable and only symptomatic treatment is offered to patients, many drugs are currently in testing with the aim to reverse the disease or to slow its progress (Kumar et al., 2015). The pathogenesis of polyQ diseases is caused by the disruption of cellular pathways by the expression products of the mutant gene, i.e., proteins containing polyQ tracts and mutant transcripts. The pathology develops in the brain areas characteristic for each disorder, e.g. striatum in HD, cerebellum in SCA3 (Fiszer \& Krzyzosiak, 2013; Evers et al., 2013; Labbadia \& Morimoto, 2013). The most direct and beneficial therapeutic strategy for polyQ diseases is silencing the causal gene expression, and the prevailing strategy is transcript targeting using ON-based tools (Bennett \& Swayze, 2010; Sibley et al., 2010; Kole et al., 2012). For several polyQ diseases, allele-selective strategies using ONs targeting regions containing SNP variants or expanded CAG repeat tracts, have been considered (Fiszer \& Krzyzosiak, 2014).

Short-interfering RNAs (siRNAs) are common tools used for post-transcriptional gene silencing in potential therapeutic strategies. In the RNAi pathway, one strand from the siRNA duplex, the guide strand, is loaded into RNA-induced silencing complex (RISC) with the core Argonaute 2 (AGO2) protein. The use of single-stranded siRNAs (guide-only siRNAs) is desired due to potentially lower off-target effects, but it was initially considered invalid due to the requirement for $\mathrm{ON}$ delivery to cells at very high concentrations (Holen et al., 2003). In cells, AGO2 loading is generally less efficient for single-strands than it is for duplexes (Martinez et al., 2002; Xu et al., 2004). A specific chemical modification pattern enables high activity of guide-only siRNAs and miRNAs (Haringsma et al., 2012; Chorn et al., 2012; Lima et al., 2012). A variety of ON chemical modifications is mainly introduced in the internucleotide bond (e.g., phosphothioate - PS) or sugar unit (e.g., 2'-O-methyl - 2'OMe, 2'-Omethoxyethyl $\left.-2^{\prime} \mathrm{OMOE}, 2^{\prime} \mathrm{F}\right)$. The efficiency of siRNAs has significantly increased as a result of their appropriate chemical modification (Amarzguioui et al., 2003; Sipa et al., 2007; Bramsen et al., 2009; Engels, 2013). Another option for activating guide-only siRNAs in cells is to design antisense strands that are able to form a self-duplex (Hossbach et al., 2006; Fiszer et al., 2013).

CAG repeat-targeting strategy, developed for several polyQ disorders, was proven effective and allele-selective in studies carried out by David Corey's group ( $\mathrm{Hu}$ et al., 2009; Hu et al., 2010; J Hu et al., 2011; Yu et al., 2012; Aiba et al., 2013; Liu et al., 2013) and by us (Fiszer et al., 2011; Fiszer et al., 2012; Fiszer et al., 2013). The CAG repeat-targeting approach is based on multiple binding sites in the expanded repeat tract, and the degree of selectivity depends on the difference between the repeat tract length in the normal and mutant alleles. In this approach, miRNA-like siRNAs are designed to contain base substitutions resulting in the formation of mis-

e-mail: A.F. agaban@ibch.poznan.pl, W.J.K. wlodkrzy@ibch. poznan.pl

Abbreviations: DRPLA (dentatorubral-pallidoluysian atrophy), HD (Huntington's disease), ONs (oligonucleotides), polyQ (polyglutamine), sd-siRNAs (self-duplexing siRNAs), PS (phosphothioate modification), RISC (RNA-induced silencing complex), SCA3 (spinocerebellar ataxia type 3), ss-siRNAs (single-stranded siRNAs), 2'OMe (2'-O-methyl modification), 2'F (2'-fluoro modification) 
matches with the target (i.e., an expanded CAG repeat tract). Different types of siRNAs have been used: siRNA duplexes (Hu et al., 2010; Fiszer et al., 2011; Hu et al., 2011; Liu et al., 2013; Hu et al., 2014a), chemically modified single-stranded siRNAs (ss-siRNAs) (Yu et al., 2012; Aiba et al., 2013; Liu et al., 2013; Hu et al., 2014b) and self-duplexing siRNAs (sd-siRNAs) (Fiszer et al., 2013).

In this study, our aim was to further consolidate the beneficial effects induced by siRNAs by modifying siRNA strands with both, nucleotide substitutions and chemical modifications. These CAG repeat-targeting siRNAs were tested in cellular models of polyQ diseases.

\section{MATERIALS AND METHODS}

Cell lines. Fibroblasts from patients with polyQ diseases (HD: GM04281 - 17/68 CAG in HTT gene, SCA3: GM06153 - 18/69 CAG in ATXN3 gene, DRPLA: GM13716 - 16/68 CAG in ATN1 gene), obtained from the Coriell Cell Repositories, were grown in a minimal essential medium (Lonza) supplemented with $8-15 \%$ fetal bovine serum (Sigma-Aldrich), antibiotics (Sigma-Aldrich) and non-essential amino acids (Sigma-Aldrich); the incubator conditions included $5 \%$ $\mathrm{CO}$ and a temperature of $37^{\circ} \mathrm{C}$. Mouse striatal cell lines (STHdh) (Trettel et al., 2000), were purchased from the Coriell Cell Repositories and grown in a medium containing DMEM (Gibco), FBS (Sigma-Aldrich), G418 and penicillin/streptomycin, with incubator conditions of $5 \%$ $\mathrm{CO}_{2}$ and $33^{\circ} \mathrm{C}$. For storage in liquid nitrogen, these cell lines were cryopreserved in the Recovery Cell Culture Freezing Medium (Gibco).

Oligonucleotides and cell transfection. RNA ONs and chemically modified ONs were synthesized by $\mathrm{Fu}-$ tureSynthesis or IDT. The sequences of oligonucleotides used in this study are presented in Fig. 1. Cell transfections were performed using Lipofectamine 2000 (Invitrogen), according to the manufacturer's instructions. Transfection efficiency was monitored using a BlockIT fluorescent siRNA (Invitrogen).

Western blot. Western blots for huntingtin and ataxin-3 proteins were performed as described previously (Fiszer et al., 2011). A western blot protocol for atrophin-1 protein analysis was adapted from (Hu et al., 2014a). Briefly, $25 \mu \mathrm{g}$ of the total protein was run on a Tris-HCl SDS-polyacrylamide gel (4\% stacking gel, 6\% resolving gel, acrylamide:bis-acrylamide ratio $37.5: 1)$ in Laemmli buffer, at $125 \mathrm{~V}$, or NuPAGE 3-8\% Tris acetate gels (Invitrogen) in XT Tricine buffer (Bio-Rad), in an ice-water bath. The immunoreaction was performed using the following antibodies: anti-atrophin-1 (Bethyl Laboratories), anti-vinculin (Cell Signaling Technology) and anti-rabbit HRP-conjugate Jackson ImmunoResearch), and detected using the WesternBright Quantum HRP Substrate (Advansta). The protein bands were directly scanned from the membrane using a camera and were quantified using the Gel-Pro Analyzer.

Northern blot. The detection of siRNA pools present in cells after transfection was performed using northern blotting, as previously described (Fiszer et al., 2013). Briefly, total RNA $(2 \mu \mathrm{g})$ and 2 pmoles of synthetic ON (in the " $\mathrm{S}$ " lane) were resolved on a denaturing polyacrylamide gel. The RNAs were transferred onto a GeneScreen Plus hybridization membrane (PerkinElmer) using semi-dry electroblotting (Sigma-Aldrich). The membrane was probed with a specific DNA probe complementary to the A2 sequence and labeled with $\left[\gamma^{32} \mathrm{P}\right]$ ATP (5000 $\mathrm{Ci} / \mathrm{mmol}$, Hartmann Analytics). The hybridization was performed overnight at $37^{\circ} \mathrm{C}$, in a buffer containing $5 \times$ SSC, $1 \%$ SDS and $1 \mathrm{X}$ Denhardt's solution. The radioactive signals were quantified by phosphorimaging (Multi Gauge v3.0, Fujifilm).

Statistical analysis. The statistical significance of silencing was assessed using a one-sample t-test, with an arbitrary value of 1 assigned to cells treated with control siRNA (C). Selected data were compared using an unpaired t-test with Welch's correction to assess the alleleselectivity of silencing (normal vs. mutant allele silencing). Two-tailed $p$ values less than 0.05 were considered to be significant. All experiments for which statistical analyses were done, were repeated three times.

\section{RESULTS}

\section{Design of chemically modified CAG repeat-targeting ss-siRNAs}

We designed several CAG repeat-targeting ONs based on (I) the nucleotide sequences of miRNA-like siRNAs that were found to be effective and allele-selective in the silencing of mutant polyQ gene expression and (II) the chemical modification patterns that were described as being suitable for ss-siRNA activity (Fig. 1). A2F, WF and $\mathrm{PF}$ were based on the sequences of previously described RNA ONs (A2 (Fiszer et al., 2013), W13/16 (Fiszer et al., 2011) and PM3 (Hu et al., 2010), respectively) and a chemical modification pattern mainly containing 2'F and two 2'OMe nucleotides from the 3'end (Chorn et al., 2012; Haringsma et al., 2012). Additionally, the WF and PF ONs contained A-substitution, forming an additional bulge in the region of interaction with the target sequence, which has been described as potentially increasing the specificity of siRNA (Dua et al., 2011). A2M was based on both, the A2 sequence and a chemical modification pattern very close to the one developed by Ionis Pharmaceuticals (Lima et al., 2012). A 5' phosphate was included in all of the ONs because it is required for activity within RISC, whereas chemically modified ONs may not be good substrates for endogenous kinases.

\section{Silencing of different mutant polyQ genes (HTT, ATXN3, ATN1) with selected chemically modified CAG repeat- targeting siRNAs in fibroblast cell models}

The activity of a set of chemically modified ONs was initially assessed in HD fibroblasts (Fig. 2A). The ONs were transfected at $100 \mathrm{nM}$, and $72 \mathrm{~h}$ later, the cells were lysed for protein isolation and huntingtin level assessment. In this HD cellular model, ONs with chemical modifications $\mathrm{A} 2 \mathrm{~F}, \mathrm{WF}$ and $\mathrm{PF}$ were tested and did not demonstrate any significantly better silencing properties when compared to unmodified ONs A2 and W1316 (marked as "W" in the figure), which were used as a reference. Nevertheless, under the tested conditions,

\begin{tabular}{|c|c|c|}
\hline $\mathrm{A} 2 \mathrm{~F}$ & 5'P GCUGCUGCAGCUGCUGCUGCU & \multirow{4}{*}{$\begin{array}{l}\text { 5'P - 5'-Phosphorylation } \\
\text { N - 2'-Fluoro } \\
\text { N - 2'-O-Methyl } \\
{ }^{*} \text { - Phosphorothioate }\end{array}$} \\
\hline WF & 5'P CAUUGCUGCUGCUGUUGUUGCUG & \\
\hline PF & 5'P GACUGCUGCAAAUGCUGCUGCU & \\
\hline A2M & $5^{\prime} P G^{*} C^{*} U G^{*} C U^{*} G C^{*} A G^{*} C U^{*} G C^{*} U^{*} G^{*} C^{*} U^{*} G^{*} C^{*} U$ & \\
\hline
\end{tabular}

Figure 1. Nucleotide sequences and chemical modifications of the tested ONs.

Nucleotides that are base substitutions resulting in mismatch formation with the target sequence are marked in bold. An additional base substitution at position 2 is underlined. 
$\mathrm{A} 2 \mathrm{~F}$ and WF caused the huntingtin levels to decrease to $\sim 50 \%$ of the control level (Fig. 2A).

The same set of ONs was tested in SCA3 fibroblasts (Fig. 2B). A2F showed very similar efficiency and selectivity in mutant ATXN3 silencing when compared to unmodified A2, e.g., the mutant ataxin-3 level was decreased to $\sim 25 \%$ of the control level, but silencing of the normal allele was also considerable. WF's silencing properties were better than the effect observed in this model for unmodified W1316: a significant allele selectivity was observed as a consequence of the mutant protein decreasing to $\sim 40 \%$ of the control level, and no changes in normal ataxin- 3 levels were observed (Fig. 2B, left panel). A2F and A2M activity was also analyzed in more detail after transfection at 1, 5, 20 or $50 \mathrm{nM}$ (Fig. 2B, right panel). Both ONs caused a significant downregulation of mutant ataxin-3, already at $5 \mathrm{nM}$. A2F showed better allele-selective properties, but A2M also caused alterations in splicing of ATXN3 transcript, and as a result a lower molecular weight protein product, lacking the exon containing polyQ tract, is produced in SCA3 cells (Liu et al., 2013).
The activity of $\mathrm{A} 2 \mathrm{~F}$ and $\mathrm{A} 2 \mathrm{M}$ was also analyzed in a DRPLA fibroblast model (Fig. 2C). Both ONs silenced the expression of ATN1 efficiently, causing atrophin-1 downregulation to $\sim 25 \%$ of the control level after transfection at $50 \mathrm{nM}$ concentration. A2F showed better allele-selective properties, as $60 \%$ of the normal protein level remained in the cells (and less than 50\% using A2M) (Fig. 2C).

\section{Evaluation of desired properties of chemically modified siRNAs}

Taking A2 and A2F ONs as an example, we wanted to verify how chemical modifications, mainly 2'F, influence ON stability in fibroblast cell culture. We transfected fibroblast cell line SCA3 with $50 \mathrm{nM}$ A2 or A2F, and isolated total RNA and protein at specific time points. We then performed northern blotting with an A2-specific probe to detect transfected ONs (Fig. 3A). A2 and A2F displayed a similar pattern of detection with a gradual decrease in their levels at the analyzed time points. It is worth emphasizing that northern blots show the total pool of ONs present in cells, which does not directly

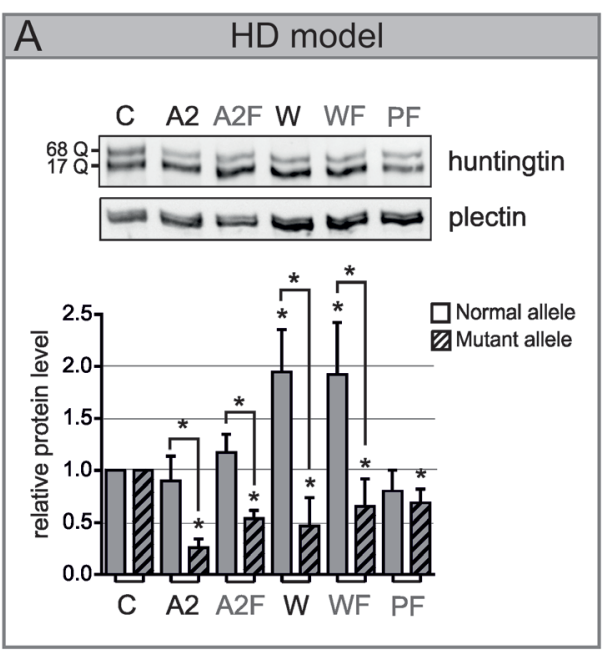

B

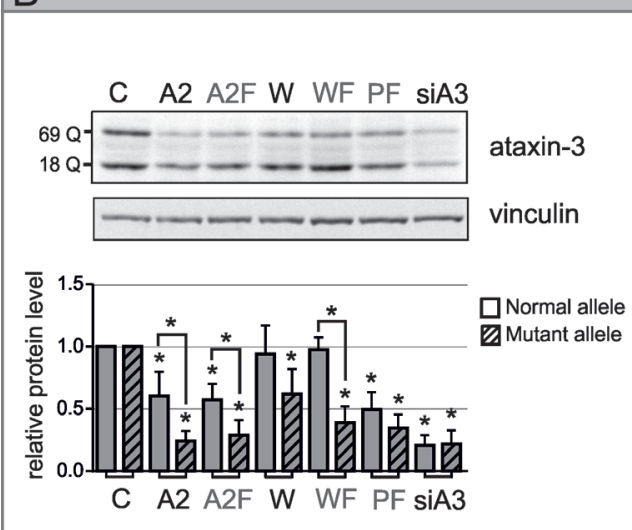

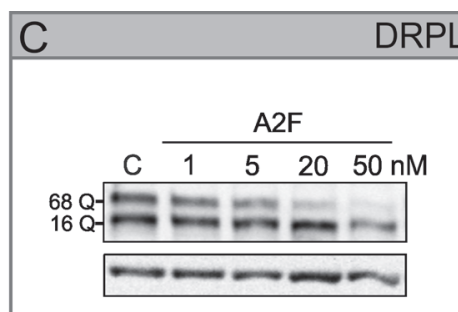
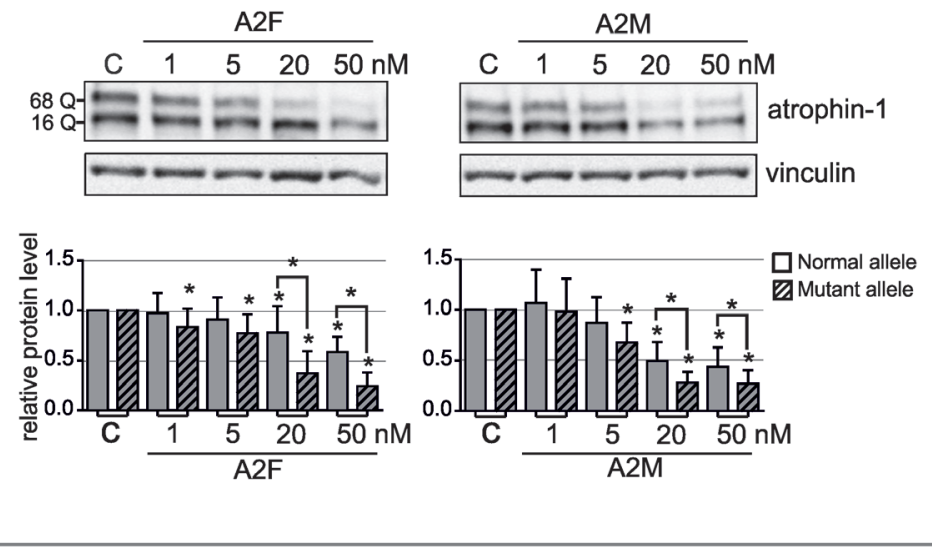

\section{SCA3 model}
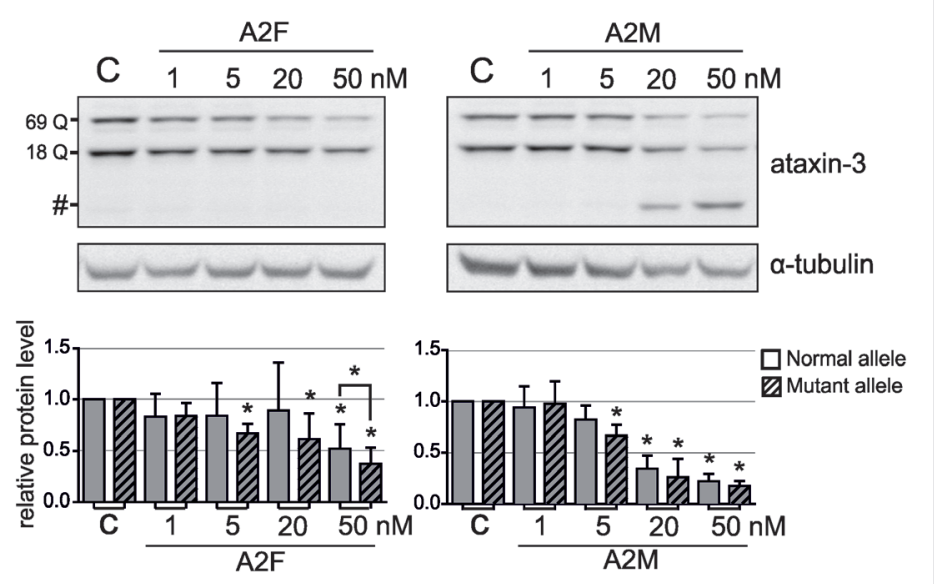

Figure 2. Efficiency and selectivity of selected ONs assessed in human fibroblasts by western blot analysis of polyQ protein levels. (A) Western blot analysis of huntingtin levels in HD fibroblasts (GM04281, 17/68 Q), at $72 \mathrm{~h}$ after transfection with $200 \mathrm{nM}$ of the indicated ON. (B) Western blot analysis of ataxin-3 levels in SCA3 fibroblasts (GM06153, 18/69 Q), at $72 \mathrm{~h}$ after transfection with $100 \mathrm{nM}$ sdsiRNA (left panel), and after transfection with 1,5, 20 or $50 \mathrm{nM}$ ON A2F or A2M (right panels) "\#" - additional ataxin-3 protein product. (C) Western blot analysis of atrophin-1 levels in DRPLA fibroblasts (GM13716, 16/68 Q), at $48 \mathrm{~h}$ after transfection with 1, 5, 20 or $50 \mathrm{nM}$ ON A2F or $\mathrm{A} 2 \mathrm{M}$; " $\mathrm{C}$ " - control line, total protein from fibroblasts transfected with BlocklT siRNA. For semi-quantitation, signal intensities were normalized to reference protein levels: plectin, a-tubulin or vinculin. The error bars represent standard deviations. The $p$-value is indicated with an asterisk $\left.{ }^{*} p<0.05\right)$. 
A
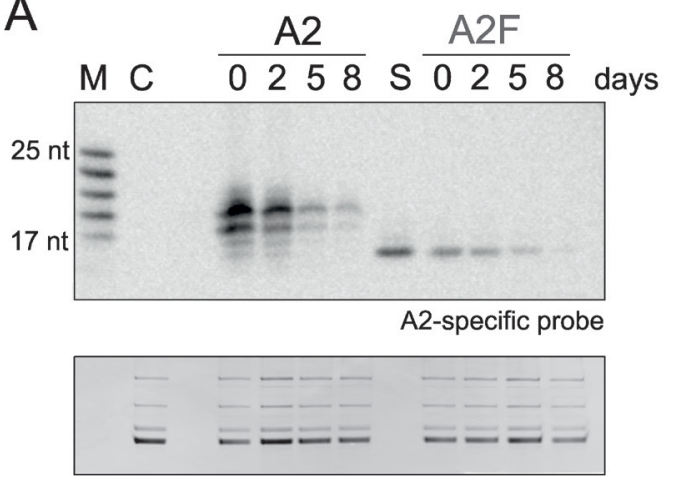

$\mathrm{EtBr}$

B
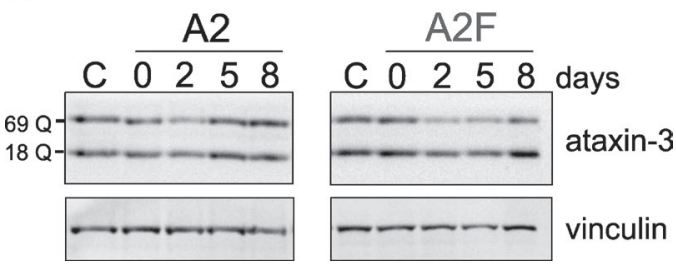

Figure 3. Time span of ON stability and silencing effects.

(A) Northern blot analysis of cellular levels of A2 and A2F. ONs were detected in the total RNA fraction from SCA3 fibroblasts (GM06153) lysed at different time points: $0,2,5$ and 8 days posttransfection, with $50 \mathrm{nM}$ ONs. Ethidium bromide (EtBr) staining was used as a loading control. " 0 " $-3 \mathrm{~h}$ post-transfection, "S" - synthetic A2F loading, " $C$ " - control line, total RNA from fibroblasts transfected with BlockIT siRNA. (B) Western blot analysis of ataxin-3 levels after the same transfections as in (A). Representative blots are shown. " $\mathrm{C}$ " - control line, total protein from fibroblasts transfected with BlockIT siRNA.

correspond to the active pool, functioning within RISC. After 8 days, the signals were very weak, and these results are in agreement with the western blot analysis, where the most significant downregulation of ataxin-3 was observed on the second day and was later less prominent (Fig. 3B). In both cases, the cells are growing during the analyzed time post-transfection, so the effect of $\mathrm{ON}$ concentration in a single cell is somewhat diluted. Because neurons are not dividing cells, in this cell type, the stability of an $\mathrm{ON}$ and the duration of its silencing effects are only dependent on its resistance to degradation.

\section{Silencing of different mutant HTT gene with selected chemically modified CAG repeat-targeting siRNAs in StHdh striatal cells}

Cultured neuronal cells are the preferred model for testing ONs because they better represent brain cells, which should be the main cell target in therapeutic treatment for polyQ diseases. The relevant neuronal cells may be derived from brain tissue of polyQ rodent models or differentiated from induced human pluripotent stem cells (iPSCs). We have used mouse striatal cell lines (Trettel et al., 2000) as a model that is more relevant to HD. First, we optimized transfection in these cells to efficiently deliver ONs (Supplementary Fig. 1 at www.actabp.pl). To evaluate mutant $H t t$ silencing, we used two cell lines, heterozygous STHdh 7/111Q and homozygous STHdh 111/111Q, and selected ONs, A2, A2F and WF, were transfected at $100 \mathrm{nM}$ concentration (Fig. 4). After $72 \mathrm{~h}$, the cells were lysed, and the huntingtin levels were assessed using western blot. All of the tested ONs silenced the expression of the Htt mutant allele encoding 111 Qs

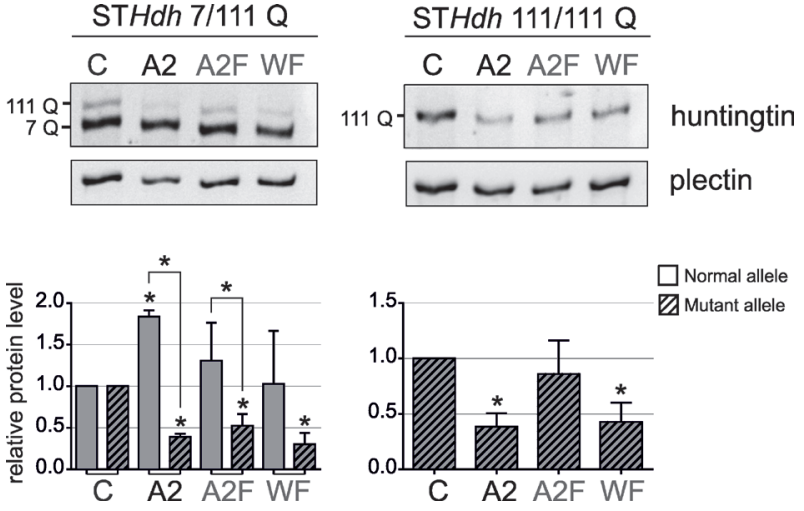

Figure 4. Western blot analysis of huntingtin levels in mouse striatal cells derived from an HD model (STHdh 7/111Q and STHdh 111/111Q) at $72 \mathrm{~h}$ after transfection with $100 \mathrm{nM}$ ONs: $\mathrm{A2}$, A2F and WF.

" $\mathrm{C}^{\prime \prime}$ - control line, total protein from fibroblasts transfected with BlockIT siRNA. For semi-quantitation, signal intensities were normalized to plectin protein levels. The error bars represent standard deviations. The $p$-value is indicated with an asterisk $\left({ }^{*} p<0.05\right)$.

in the heterozygous cell line, with protein downregulation reaching $30 \%$ of the control level. The expression of the normal allele encoding 7 Qs remained at the control level or was upregulated by A2. In the homozygous cell line containing two mutant Htt alleles encoding 111 Qs, A2 and WF showed an efficient downregulation of huntingtin, reaching $40-45 \%$ of the control level (Fig. 4).

\section{DISCUSSION}

Currently, two strategies are being tested to ensure a long-lasting activity of ONs: (I) the incorporation of chemical modifications to increase $\mathrm{ON}$ stability and (II) delivery of silencing reagents in genetic vectors. These strategies differ in the design of the relevant therapeutic tools and in the obstacles that need to be overcome in preclinical testing. The drawbacks of genetic vectors are as follows: applicability to RNAi tools only, problematic dosage control, and possible mutagenicity and immunogenicity of the viral vectors. Synthetic ONs offer an exciting alternative because both, the sequence and the chemical modification pattern of the ON can be optimized to achieve high in vivo efficiency together with low toxicity. Synthetic ONs may require repetitive administration, but their transient activity may be considered an advantage with respect to safety owing to direct dosage control. In addition, synthetic ONs have been found to be more equally and broadly dispersed in the brain because free uptake by neuronal cells is observed for ONs delivered via an intra-CNS route. In several cases of ONs testing for polyQ diseases, synthetic siRNAs or AONs were either locally injected or infused into the CNS, which caused an efficient downregulation of the targeted gene (Wang et al., 2005; DiFiglia et al., 2007; Stiles et al., 2011; Kordasiewicz et al., 2012; Yu et al., 2012; Ostergaard et al., 2013).

An important aspect of ON design is the selection of a chemical modification pattern that is suitable for its in vivo activity. Chemical modification not only increases the biological stability of the $\mathrm{ON}$ and the efficiency of its hybridization with the target sequence, but may also be critical for its effective delivery and low toxicity. The PS, 2'F and 2'OMe modifications are well tolerated by RISC. Additionally, the use of one strand of siRNA may 
be considered more straightforward and safer due to the lack of potential unwanted activity of a sense strand.

We designed RNAi-based ONs that contained specific base substitutions and chemical modification patterns. By these means, we wanted to achieve an effective and specific silencing of selected mutant genes. Generally, the activity of the tested oligonucleotides was not significantly changed when compared to the pure RNA versions. Nevertheless, the use of chemically modified versions is expected to be more beneficial in in vivo experiments conducted in mouse models. We also tested a modified version of sd-siRNA A6, which originally did not induce silencing of HTT expression (Fiszer et al., 2013). Its modified version, A6M (analogous chemical modification pattern to A2M), was also inactive (data not shown), which implicates the dominant role of basesubstitution patterns for activity of this type of ONs.

At this point, it is difficult to say which ON-based therapeutic strategy is best suited for polyQ diseases. The existing strategies offer great opportunities for therapy but also have limitations. The allele-selective strategies are considered to be safer. The CAG repeat-targeting strategy, which was considered some years ago to be a "mission impossible", turned out to be not only feasible, but also very promising according to the first in vivo studies in mouse models of HD ( $\mathrm{Yu}$ et al., 2012). The potential advantage of this strategy is its universal scope as one drug could possibly be used to treat most of the polyQ disorders.

\section{Acknowledgements}

The authors would like to thank Agnieshka Agasing and Ewa Dzwonkowska for their participation in the project.

This work was supported by a grant from the Polish Ministry of Science and Higher Education (IP2012032472 to A.F.), the National Science Centre, Poland $(2015 / 17 / D / N Z 5 / 03443$ to A.F. and 2014/15/B/ NZ1/01880 to W.J.K.), and by the quality-promoting subsidy, under the Leading National Research Centre (KNOW) program for the years 2014-2019. A.F. is a holder of a scholarship from the Polish Ministry of Science and Higher Education. The authors declare no conflict of interest.

\section{REFERENCES}

Aiba Y, Hu J, Liu J, Xiang Q, Martinez C, Corey DR (2013) Alleleselective inhibition of huntingtin and ataxin-3 expression by RNA duplexes containing unlocked nucleic acid (UNA) substitutions. Biochemistry 52: 9329-9338. doi:10.1021/bi4014209

Amarzguioui M, Holen T, Babaie E, Prydz H (2003) Tolerance for mutations and chemical modifications in a siRNA. Nucleic Acids Res 31: 589-595. doi:10.1093/nar/gkg147

Bennett CF, Swayze EE. (2010) RNA targeting therapeutics: molecular mechanisms of antisense oligonucleotides as a therapeutic platform. Annu Rev Pharmacol Toxicol 50: 259-293. doi:10.1146/annurev. pharmtox.010909.105654

Bramsen JB, Laursen MB, Nielsen AF, Hansen TB, Bus C, Langkjaer N, Babu BR, Højland T, Abramov M, Van Aerschot A, Odadzic D, Smicius R, Haas J, Andree C, Barman J, Wenska M, Srivastava $P$, Zhou C, Honcharenko D, Hess S, Müller E, Bobkov GV, Mikhailov SN, Fava E, Meyer TF, Chattopadhyaya J, Zerial M, Engels JW, Herdewijn P, Wengel J, Kjems J (2009) A large-scale chemical modification screen identifies design rules to generate siRNAs with high activity, high stability and low toxicity. Nucleic Acids Res 37: 2867-2881. doi:10.1093/nar/gkp106

Chorn G, Klein-McDowell M, Zhao L, Saunders MA, Flanagan WM, Willingham AT, Lim LP (2012) Single-stranded microRNA mimics. RNA 18: 1796-1804. doi:10.1261/rna.031278.111

DiFiglia M, Sena-Esteves M, Chase K, Sapp E, Pfister E, Sass M, Yoder J, Reeves P, Pandey RK, Rajeev KG, Manoharan M, Sah DW, Zamore PD, Aronin N (2007) Therapeutic silencing of mutant hun- tingtin with siRNA attenuates striatal and cortical neuropathology and behavioral deficits. Proc Natl Acad Sci U S A 104: 17204-17209 Dua P, Yoo JW, Kim S, Lee DK (2011) Modified siRNA structure with a single nucleotide bulge overcomes conventional siRNAmediated off-target silencing. Mol Ther 19: 1676-1687. doi:10.1038/ mt.2011.109

Engels JW (2013) Gene silencing by chemically modified siRNAs. N Biotechnol 30: 302-307. doi:10.1016/j.nbt.2012.07.002.

Evers MM, Toonen LJ, van Roon-Mom WM (2013) Ataxin-3 protein and RNA toxicity in spinocerebellar ataxia type 3: current insights and emerging therapeutic strategies. Mol Neurobiol 49: 1513-1531. doi:10.1007/s12035-013-8596-2

Fan HC, Ho LI, Chi CS, Chen SJ, Peng GS, Chan TM, Lin SZ, Harn HJ (2014) Polyglutamine (polyQ) diseases: genetics to treatments. Cell Transplant 23: 441-458. doi:10.3727/096368914X678454

Fiszer A, Krzyzosiak WJ (2013) RNA toxicity in polyglutamine disorders: concepts, models, and progress of research. J Mol Med (Berl) 91: 683-691. doi:10.1007/s00109-013-1016-2

Fiszer A, Krzyzosiak WJ (2014) Oligonucleotide-based strategies to combat polyglutamine diseases. Nucleic Acids Res 42: 6787-810. doi:10.1093/nar/gku385

Fiszer A, Mykowska A, Krzyzosiak WJ (2011) Inhibition of mutant huntingtin expression by RNA duplex targeting expanded CAG repeats. Nucleic Acids Res 39: 5578-5585. doi: 10.1093/nar/gkr156

Fiszer A, Olejniczak M, Galka-Marciniak P, Mykowska A, Krzyzosiak WJ (2013) Self-duplexing CUG repeats selectively inhibit mutant huntingtin expression. Nucleic Acids Res 41: 10426-10437. doi: $10.1093 /$ nar/gkt825

Fiszer A, Olejniczak M, Switonski PM, Wroblewska JP, WisniewskaKruk J, Mykowska A, Krzyzosiak WJ (2012) An evaluation of oligonucleotide-based therapeutic strategies for polyQ diseases. BMC Mol Biol 13: 6. doi:10.1186/1471-2199-13-6

Haringsma HJ, Li JJ, Soriano F, Kenski DM, Flanagan WM, Willingham AT (2012) mRNA knockdown by single strand RNA is improved by chemical modifications. Nucleic Acids Res 40: 4125-4136. doi:10.1093/nar/gkr1301.

Holen T, Amarzguioui M, Babaie E, Prydz H (2003) Similar behaviour of single-strand and double-strand siRNAs suggests they act through a common RNAi pathway. Nucleic Acids Res 31: 2401-2407. doi:10.1093/nar/gkg338

Hossbach M, Gruber J, Osborn M, Weber K, Tuschl T (2006) Gene silencing with siRNA duplexes composed of target-mRNA-complementary and partially palindromic or partially complementary single-stranded siRNAs. RNA Biol 3: 82-89.

Hu J, Gagnon KT, Liu J, Watts JK, Syeda-Nawaz J, Bennett CF, Swayze EE, Randolph J, Chattopadhyaya J, Corey DR (2011) Allele-selective inhibition of ataxin-3 (ATX3) expression by antisense oligomers and duplex RNAs. Biol Chem 392: 315-325. doi: 10.1515/ BC. 2011.045

Hu J, Liu J, Corey DR (2010) Allele-selective inhibition of huntingtin expression by switching to an miRNA-like RNAi mechanism. Chem Biol 17: 1183-1188. doi: 10.1016/j.chembiol.2010.10.013

Hu J, Liu J, Narayanannair KJ, Lackey JG, Kuchimanchi S, Rajeev KG, Manoharan M, Swayze EE, Lima WF, Prakash TP, Xiang Q, Martinez C, Corey DR (2014a) Allele-selective inhibition of mutant atrophin-1 expression by duplex and single-stranded RNAs. Biochemistry 53: 4510-4518. doi:10.1021/bi500610r.

Hu J, Liu J, Yu D, Aiba Y, Lee S, Pendergraff H, Boubaker J, Artates JW, Lagier-Tourenne C, Lima WF, Swayze EE, Prakash TP, Corey DR (2014b) Exploring the effect of sequence length and composition on allele-selective inhibition of human huntingtin expression by single-stranded silencing RNAs. Nucleic Acid Ther 24: 199-209. doi:10.1089/nat.2013.0476

Hu J, Matsui M, Gagnon KT, Schwartz JC, Gabillet S, Arar K, Wu J, Bezprozvanny I, Corey DR (2009) Allele-specific silencing of mutant huntingtin and ataxin-3 genes by targeting expanded CAG repeats in mRNAs. Nat Biotechnol 27: 478-484. doi:10.1038/nbt.1539

Kole R, Krainer AR, Altman S (2012) RNA therapeutics: beyond RNA interference and antisense oligonucleotides. Nat Rev Drug Discov 11: 125-140. doi: 10.1038/nrd3625

Kordasiewicz HB, Stanek LM, Wancewicz EV, Mazur C, McAlonis MM, Pytel KA, Artates JW, Weiss A, Cheng SH, Shihabuddin LS, Hung G, Bennett CF, Cleveland DW (2012) Sustained therapeutic reversal of Huntington's disease by transient repression of huntingtin synthesis. Neuron 74: 1031-1044. doi:10.1016/j.neuron.2012.05.009

Kumar A, Kumar Singh S, Kumar V, Kumar D, Agarwal S, Rana MK (2015) "Huntington's disease: an update of therapeutic strategies. Gene 556: 91-97. doi:10.1016/j.gene.2014.11.022

Labbadia J, Morimoto RI (2013) Huntington's disease: underlying molecular mechanisms and emerging concepts. Trends Biochem Sci 38: 378-385. doi:10.1016/j.tibs.2013.05.003

Lima WF, Prakash TP, Murray HM, Kinberger GA, Li W, Chappell AE, Li CS, Murray SF, Gaus H, Seth PP, Swayze EE, Crooke ST (2012) Single-stranded siRNAs activate RNAi in animals. Cell 150: 883-894. doi:10.1016/j.cell.2012.08.014 
Liu J, Pendergraff H, Narayanannair KJ, Lackey JG, Kuchimanchi S, Rajeev KG, Manoharan M, Hu J, Corey DR (2013) RNA duplexes with abasic substitutions are potent and allele-selective inhibitors of huntingtin and ataxin-3 expression. Nucleic Acids Res 41: 8788-8801. doi:10.1093/nar/gkt594

Liu J, Yu D, Aiba Y, Pendergraff H, Swayze EE, Lima WF, Hu J, Prakash TP, Corey DR (2013) ss-siRNAs allele selectively inhibit ataxin-3 expression: multiple mechanisms for an alternative gene silencing strategy. Nucleic Acids Res 41: 9570-9583. doi:10.1093/nar/ gkt693

Martinez J, Patkaniowska A, Urlaub H, Lührmann R, Tuschl T (2002) Single-stranded antisense siRNAs guide target RNA cleavage in RNAi. Cell 110: 563-574.

Østergaard ME, Southwell AL, Kordasiewicz H, Watt AT, Skotte NH, Doty CN, Vaid K, Villanueva EB, Swayze EE, Bennett CF, Hayden MR, Seth PP (2013) Rational design of antisense oligonucleotides targeting single nucleotide polymorphisms for potent and allele selective suppression of mutant Huntingtin in the CNS. Nucleic Acids Res 41: 9634-9650. doi:10.1093/nar/gkt725

Sibley CR, Seow Y, Wood MJ (2010) Novel RNA-based strategies for therapeutic gene silencing. Mol Ther 18: 466-476. doi:10.1038/ mt.2009.306

Sipa K, Sochacka E, Kazmierczak-Baranska J, Maszewska M, Janicka M, Nowak G, Nawrot B (2007) Effect of base modifications on structure, thermodynamic stability, and gene silencing activi- ty of short interfering RNA. RNA 13: 1301-1316. doi:10.1261/ rna.538907

Stiles DK, Zhang Z, Ge P, Nelson B, Grondin R, Ai Y, Hardy P, Nelson PT, Guzaev AP, Butt MT, Charisse K, Kosovrasti V, Tchangov L, Meys M, Maier M, Nechev L, Manoharan M, Kaemmerer WF, Gwost D, Stewart GR, Gash DM, Sah DW (2011) Widespread suppression of huntingtin with convection-enhanced delivery of siRNA. Exp Neurol 233: 463-471. doi:10.1016/j.expneurol.2011.11.020

Trettel F, Rigamonti D, Hilditch-Maguire P, Wheeler VC, Sharp AH, Persichetti F, Cattaneo E, MacDonald ME (2000) Dominant phenotypes produced by the HD mutation in STHdh(Q111) striatal cells. Hum Mol Genet 9: 2799-2809.

Wang YL, Liu W, Wada E, Murata M, Wada K, Kanazawa I (2005) Clinico-pathological rescue of a model mouse of Huntington's disease by siRNA. Neurosci Res 53: 241-249.

Xu Y, Linde A, Larsson O, Thormeyer D, Elmen J, Wahlestedt C, Liang Z (2004) Functional comparison of single- and double-stranded siRNAs in mammalian cells. Biochem Biophys Res Commun 316: 680-687.

Yu D, Pendergraff H, Liu J, Kordasiewicz HB, Cleveland DW, Swayze EE, Lima WF, Crooke ST, Prakash TP, Corey DR (2012) Single-stranded RNAs use RNAi to potently and allele-selectively inhibit mutant huntingtin expression. Cell 150: 895-908. doi:10.1016/j. cell.2012.08.002 